\title{
Detection of circulating tumor cells by reverse transcription-quantitative polymerase chain reaction and magnetic activated cell sorting in the peripheral blood of patients with hepatocellular carcinoma
}

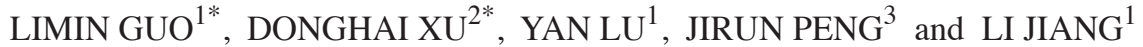 \\ Departments of ${ }^{1}$ Hepatobiliary Surgery and ${ }^{2}$ Radiology, Beijing Ditan Hospital, Capital Medical University, Beijing 100015; \\ ${ }^{3}$ Department of Surgery, Beijing Shijitan Hospital, Capital Medical University, Beijing 100038, P.R. China
}

Received February 28, 2016; Accepted February 23, 2017

DOI: $10.3892 / \mathrm{mmr} .2017 .7372$

\begin{abstract}
Hepatocellular carcinoma (HCC) is one of the most lethal malignancies worldwide. Circulating tumor cells (CTCs) are considered a major cause of recurrence and metastasis in cancer; however, the detection of CTCs is challenging owing to their very low numbers in peripheral blood (around $10 \mathrm{CTCs}$ per 1,000,000 erythrocytes). Cancer-testis antigens (CTAs) are specific tumor markers for CTCs. The present study aimed to evaluate the sensitivity and specificity of reverse transcription-quantitative polymerase chain reaction (RT-qPCR) for the detection of nine CTAs as well as placenta-specific antigen 1 (PLAC1) in peripheral blood mononuclear cell (PBMC) samples collected from 51 patients with HCC. The effectiveness of magnetic-activated cell sorting (MACS) for tumor-cell enrichment, through the depletion of $\mathrm{CD} 45^{+}$leukocytes in PBMC samples, was also assessed. Immunocytochemistry along with hematoxylin and eosin staining demonstrated that RT-qPCR achieved an overall positive detection rate for CTAs and PLAC1 of $70.6 \%$; the highest rates were observed for melanoma-associated antigen A3 (MAGEA3), synovial sarcoma X breakpoint 1, MAGEA1, NY-ESO-1, L antigen 1 and PLAC1. MACS-detected intact CTCs in PBMCs were confirmed by $\mathrm{H} \& \mathrm{E}$ staining and morphological assessment; 12 out of $19(63.2 \%)$ patients were identified as positive for CTAs. Screening for these five CTAs and PLAC1 by RT-qPCR may offer a potentially valuable prognostic tool with good
\end{abstract}

Correspondence to: Professor Li Jiang, Department of Hepatobiliary Surgery, Beijing Ditan Hospital, Capital Medical University, 8 Jingshun East Street, Chaoyang, Beijing 100015, P.R. China

E-mail: jiangli1903@163.com

*Contributed equally

Key words: hepatocellular carcinoma, circulating tumor cells, reverse transcription-quantitative polymerase chain reaction, immunomagnetic beads, tumor cell enrichment sensitivity and specificity in patients with HCC that may be enhanced by MACS.

\section{Introduction}

Hepatocellular carcinoma (HCC) is one of the most lethal malignancies worldwide, and is the third most common cause of cancer-associated mortality. Previous epidemiological studies revealed that $\sim 626,000$ new diagnoses of HCC are made annually (1). Despite improved therapeutic modalities, the prognosis for patients with HCC remains poor, primarily owing to difficulties in early diagnosis and the high rates of recurrence and metastasis following curative resection; $54-62 \%$ of patients develop recurrence within 5 years of surgery, and the overall 5-year survival rate of patients with HCC is $<20 \%$ (2).

Circulating tumor cells (CTCs) are tumor cells that are found in the peripheral blood of patients with malignant disease, and are increasingly being recognized as a major cause of recurrence and metastasis (3). Therefore, early detection of CTCs may facilitate prognostic evaluations and contribute to effective therapeutic regimens in patients with HCC. However, the number of CTCs in peripheral blood is extremely low, with only a few cells/ml, compared with the billions of leukocytes and erythrocytes that may be found (4). Advances in molecular technologies and the discovery of novel tumor markers are making it possible to detect these rare cells with higher sensitivity and specificity. Although reverse transcription-polymerase chain reaction (RT-PCR) and nested-primer RT-PCR may be very sensitive, they sometimes yield false positive results (5). By contrast, quantitative RT-PCR (RT-qPCR) may measure very low expression levels of tumor marker genes with precise and reproducible results, and therefore may be a powerful tool to detect CTCs in peripheral blood mononuclear cell (PBMC) samples (6).

Albumin and $\alpha$-fetoprotein (AFP) are the most commonly used tumor markers for the detection of CTCs in patients with HCC. However, they are non-specific and may occasionally be observed in the peripheral blood of patients with nonmalignant liver diseases, including cirrhosis or hepatitis. Previous reports have revealed that the positive detection 
rates for these markers using RT-PCR or nested RT-PCR range between 23.4 and $72.1 \%$ (7,8). Cancer-testis antigens (CTAs) have recently been identified as specific tumor markers for CTCs $(9,10)$. CTAs belong to a superfamily of proteins that are solely expressed in tumors and in the testes, but not in normal tissues $(11,12)$. Members of this superfamily include: Fetal and adult testis-expressed 1 (FATE1), melanoma-associated antigen C2 (MAGEC2), L antigen 1 (LAGE1), MAGEA1, MAGEA3, NY-ESO-1, sperm protein associated with the nucleus on the $\mathrm{X}$ chromosome (SPANX) A1, SPANXC and synovial sarcoma $\mathrm{X}$ breakpoint 1 (SSX1). Placenta-specific antigen 1 (PLAC1) exhibits a similar expression pattern as CTAs, and is expressed in a variety of tumors and in the placenta, but rarely in normal tissues (13).

In the present study, the effectiveness of RT-qPCR for detecting CTCs in patients with HCC was assessed by evaluating the mRNA expression levels of nine CTAs and PLAC1 in PBMC samples from 51 preoperative patients with HCC. In addition, immunomagnetic beads were used to enrich the PBMC samples for tumor cells to identify the presence of CTCs morphologically. By using RT-qPCR, CTCs were detected in $\sim 70 \%$ of patients with HCC, indicating that this technique may by enhanced by magnetic-activated cell sorting (MACS).

\section{Materials and methods}

Ethical approval and informed consent. The experiments of the present study were approved by the ethics committee of Beijing Ditan Hospital (Capital Medical University, Beijing, China) and written informed consent was obtained from patients prior to enrollment in the study.

Cell lines and cell culture. The QGY7703 human HCC cell line was used in the present study as it expresses the nine target CTAs and PLAC1 genes at moderate levels, as determined by our preliminary experiment (data not shown). The QGY7703 cell line was purchased from the Institute of Biochemistry and Cell Biology (Shanghai, China). Cells were cultured in RPMI-1640 medium (Gibco; Thermo Fisher Scientific, Inc., Waltham, MA, USA) supplemented with $10 \%$ fetal calf serum (FBS; Hyclone; GE Healthcare Life Sciences, Logan, UT, USA), $2 \mathrm{mmol}$ glutamine and antibiotics (penicillin $100 \mathrm{U} / \mathrm{ml}$ and streptomycin $100 \mu \mathrm{g} / \mathrm{ml}$ ), at $37^{\circ} \mathrm{C}$ in a humidified atmosphere containing $5 \% \mathrm{CO}_{2}$.

Patient characteristics and peripheral blood sample preparation. Peripheral blood $(10-15 \mathrm{ml})$ was collected from 51 preoperative patients with $\mathrm{HCC}, 10$ patients with liver cirrhosis and 20 healthy donors. Clinicopathological characteristics of the patients and the controls are summarized in Table I. Samples were mixed with heparin to prevent coagulation, and were diluted with $10 \mathrm{ml}$ RPMI-1640 medium supplemented with $10 \%$ FBS, 2 mmol glutamine and antibiotics. The samples were placed on a Ficoll-Hypaque layer (Thermo Fisher Scientific, Inc.) and PBMCs were collected by density gradient centrifugation at $670 \mathrm{x} \mathrm{g}$ and room temperature for $20 \mathrm{~min}$.

Total RNA extraction and cDNA synthesis. Total RNA was isolated from $1 \times 10^{7}$ cultured QGY7703 cells and PBMC samples using an RNeasy kit (Qiagen $\mathrm{GmbH}$, Hilden,
Germany) and treated with RNase-free DNase I (Qiagen $\mathrm{GmbH}$ ), following the manufacturer's protocol. First strand cDNA was reverse transcribed from total RNA $(2 \mu \mathrm{g})$ using random hexamers $\left[\mathrm{d}(\mathrm{N})_{6}\right](10 \mathrm{pmol} / \mu \mathrm{l} ; 2 \mu \mathrm{l})$ and Superscript II Reverse Transcriptase $(200 \mathrm{U} / \mu \mathrm{l} ; 1 \mu \mathrm{l})$ at $50^{\circ} \mathrm{C}$ for $50 \mathrm{~min}$ (Thermo Fisher Scientific, Inc.), according to manufacturer's protocol.

Preparation of recombinant plasmids as copy number standards for RT-qPCR. Human FATE1, MAGEC2, LAGE1, MAGEA1, MAGEA3, NY-ESO-1, SPANXA1, SPANXC and SSX1 cDNAs were amplified from human testis cDNA by qPCR; PLAC1 cDNA was amplified from placenta cDNA by PCR. Human testis (from patients with advanced prostate cancer following castration therapy) and placenta (from patients following normal delivery) samples were obtained from Beijing Ditan Hospital. Amplification reactions were carried out using cDNA $(1 \mu \mathrm{l})$, forward and reverse primers $(10 \mathrm{pM}$ each), 2X PCR Master Mix (12.5 $\mu$ l; Invitrogen; Thermo Fisher Scientific, Inc.), and double-distilled $\mathrm{H}_{2} \mathrm{O}$ to a final volume of $25 \mu \mathrm{l}$. PCR products were purified using the Wizard ${ }^{\circledR}$ SV Gel and PCR Clean-Up System (Promega Corporation, Madison, WI, USA) and cloned into a pGEM-T Easy Vector system (Promega Corporation), following the manufacturer's protocol. Recombinant plasmids were confirmed by DNA sequencing. Briefly, plasmids were linearized by digestion with an EcoRI/Nco1 restriction endonuclease at $37^{\circ} \mathrm{C}$ for $4 \mathrm{~h}$ (Takara Bio, Inc., Otsu, Japan) before being purified by $1.5 \%$ agarose gel electrophoresis and the Wizard ${ }^{\circledR}$ SV Gel and PCR Clean-Up System (Promega Corporation), and quantified using a spectrophotometer at a wavelength of $260 \mathrm{~nm}$. The plasmid copy numbers (copies $/ \mathrm{ml}$ ) were calculated using the following formula: [plasmid concentration $\left.(\mathrm{g} / \mathrm{ml}) \times 6.023 \times 10^{23}\right] /$ (number of base pairs in the plasmid $x$ 660). The number of base pairs in the plasmid includes the plasmid vector and the gene insert. These were used as the copy number standards for subsequent RT-qPCR analyses.

$R T-q P C R$ and construction of standard curves. RT-qPCR primers were designed to specifically amplify the target cDNA fragments containing at least one intron, while avoiding contamination from genomic DNA. Primer sequences and TaqMan probes are listed in Table II; the housekeeping gene GAPDH was used as an internal control. Five 10-fold dilutions of the resulting constructs, ranging from $1 \times 10^{4}$ to $1 \times 10^{8}$ copies $\mu \mathrm{l}^{-1}$, were used to construct the standard curves for GAPDH, LAGE, NY-ESO-1, MAGEA1, MAGEA3, HCA587, FATE, SPANXA1, SPANXC, PLAC1 and SSX-1. Absolute quantification of mRNA using RT-qPCR was performed, which employed standard curves for quantification (14).

TaqMan probes were labeled at the $5^{\prime}$ end with the 6-carboxyfluorescein fluorophore, and at the 3 ' end with the fluorescence quencher tetramethylrhodamine. qPCR reactions were prepared as follows: $1 \mu \mathrm{l}$ cDNA ( 100 ng total RNA) or plasmid DNA (containing $10^{4}-10^{8}$ copies of the target sequence) was mixed with $7.5 \mathrm{pM}$ of each forward and reverse primers, $6.25 \mathrm{pM}$ TaqMan probe and 2X qPCR buffer to a final volume of $25 \mu \mathrm{l}$. A positive control containing 10 copies of plasmid DNA was prepared. To test for potential contaminants, two reactions were prepared that contained either $1 \mu \mathrm{l}$ 
Table I. Clinical parameters of the 51 patients with hepatocellular carcinoma and the controls.

\begin{tabular}{|c|c|c|c|}
\hline $\begin{array}{l}\text { Clinicopathological } \\
\text { characteristics }\end{array}$ & $\begin{array}{l}\text { Hepatocellular carcinoma, } \\
\qquad \mathrm{n}=51\end{array}$ & $\begin{array}{l}\text { Cirrhosis donors, } \\
\mathrm{n}=10\end{array}$ & $\begin{array}{l}\text { Healthy donors, } \\
\qquad \mathrm{n}=20\end{array}$ \\
\hline \multicolumn{4}{|l|}{ Sex } \\
\hline Male & 35 & 7 & 13 \\
\hline Female & 16 & 3 & 7 \\
\hline \multicolumn{4}{|l|}{ Age (years) } \\
\hline$<40$ & 6 & 1 & 3 \\
\hline$\geq 40$ & 45 & 9 & 17 \\
\hline \multicolumn{4}{|l|}{ AFP (ng/ml) } \\
\hline$<400$ & 34 & - & - \\
\hline$\geq 400$ & 17 & - & - \\
\hline \multicolumn{4}{|l|}{ Tumor size (cm) } \\
\hline$<3$ & 40 & NA & NA \\
\hline$\geq 3$ & 11 & NA & NA \\
\hline \multicolumn{4}{|l|}{ Invasion of main portal vein } \\
\hline Yes & 3 & NA & NA \\
\hline No & 48 & NA & NA \\
\hline \multicolumn{4}{|l|}{ TNM stage } \\
\hline I and II & 33 & NA & NA \\
\hline III and IV & 18 & NA & NA \\
\hline \multicolumn{4}{|l|}{ Hepatitis B virus } \\
\hline Positive & 41 & 8 & 0 \\
\hline Negative & 10 & 2 & 20 \\
\hline \multicolumn{4}{|l|}{ Grade } \\
\hline Well differentiated & 6 & NA & NA \\
\hline Moderately differentiated & 38 & NA & NA \\
\hline Poorly differentiated & 7 & NA & NA \\
\hline \multicolumn{4}{|l|}{ Modus operandi } \\
\hline Excision & 46 & NA & NA \\
\hline Orthotopic liver transplantation & 5 & NA & NA \\
\hline
\end{tabular}

Levels of AFP were not measured in patients with cirrhosis or healthy donors. AFP, $\alpha$-fetoprotein; TNM, tumor-node-metastasis; NA, not applicable.

water or $1 \mu 1$ reverse transcriptase without RNA. qPCR was performed at $50^{\circ} \mathrm{C}$ for $2 \mathrm{~min}$ and $95^{\circ} \mathrm{C}$ for $10 \mathrm{~min}$, followed by 40 cycles of $92^{\circ} \mathrm{C}$ for $15 \mathrm{sec}$ and $60^{\circ} \mathrm{C}$ for $1 \mathrm{~min}$, using an ABI PRISM 7700 Sequence Detection System (Applied Biosystems; Thermo Fisher Scientific, Inc.). Each experiment was performed in triplicate.

Sensitivity of RT-qPCR and standard RT-PCR for the detection of MAGEA1 and SSX1 cDNA in different ratios of HCC cells to normal PBMC. HCC to normal PBMC ratios of $10^{-2}$, $10^{-3}, 10^{-4}, 10^{-5}$ and $10^{-6}$ were prepared by mixing $10^{5}, 10^{4}, 10^{3}$, $10^{2}$ and $10^{1}$ QGY7703 cells, respectively, with $10^{7}$ normal PBMCs from healthy individuals. A control with only normal PBMCs was additionally prepared. The cells were recovered by Ficoll-Hypaque density gradient centrifugation $(670 \mathrm{x} \mathrm{g}$ for $20 \mathrm{~min}$ at room temperature) and total RNA was extracted as aforementioned. The expression levels of MAGEA1 and SSX1 cDNA in each of the six samples were measured by RT-qPCR and standard RT-PCR. Amplification reactions for standard RT-PCR were performed using cDNA $(1 \mu \mathrm{l})$, forward and reverse primers (10 pM each), 2X PCR Master Mix (12.5 $\mu \mathrm{l}$; Thermo Fisher Scientific, Inc.) and double-distilled $\mathrm{H}_{2} \mathrm{O}$ to a final volume of $25 \mu \mathrm{l}$. The following optimal thermal cycling conditions were used: $94^{\circ} \mathrm{C}$ for $10 \mathrm{~min}$, followed by 35 cycles of $94^{\circ} \mathrm{C}$ for $25 \mathrm{sec}, 54^{\circ} \mathrm{C}$ for $25 \mathrm{sec}$ and $72^{\circ} \mathrm{C}$ for $25 \mathrm{sec}$; followed by $72^{\circ} \mathrm{C}$ for $8 \mathrm{~min}$. The housekeeping gene GAPDH was used as a control. The optimal thermal cycling conditions for GAPDH were the same for SSX1 and MAGEA1 except that the annealing temperature was $57^{\circ} \mathrm{C}$ for GAPDH. The same forward and reverse primers were used in RT-qPCR and standard RT-PCR.

Enrichment of HCC cells in PBMC samples by depletion of $C D 45^{+}$cells by MACS. PBMC samples were acquired from 19 HCC patients that were identified to have positive expression levels of the tested CTAs by RT-qPCR. PBMC samples from 10 patients with liver cirrhosis were additionally used, 
Table II. List of primer sequences and TaqMan probes.

\begin{tabular}{|c|c|c|c|}
\hline Gene & Primer sequence $\left(5^{\prime}-3^{\prime}\right)$ & $\begin{array}{l}\text { Expected } \\
\text { size (bp) }\end{array}$ & Probe $\left(5^{\prime}-3^{\prime}\right)$ \\
\hline FATE1 & $\begin{array}{l}\text { F: GCCAAGGGGAAAACCAAGAG } \\
\text { R: TCGGGTGGCAGTCATATTCC }\end{array}$ & 152 & CAAAAAGCTGCTGGCTCTGCTTCAGC \\
\hline MAGEC2 & $\begin{array}{l}\text { F: AGGGACCTCCCACCATAGAGA } \\
\text { R: CCTCCTCTGCTGTGCTGACTT }\end{array}$ & 130 & CCGGCCTGTACTGCGCTGCC \\
\hline LAGE1 & $\begin{array}{l}\text { F: CCGCCTGCTTCAGTTGC } \\
\text { R: CTGCAGCAGTCAGTCGGATA }\end{array}$ & 167 & ACGATGCCTTTCTCGTCGCCCAT \\
\hline MAGEA1 & $\begin{array}{l}\text { F: AGAGGAGCACCAAGGAGAAGA } \\
\text { R: GGCAGCAGGCAGGAGTGT }\end{array}$ & 74 & CTGCCTGTGGGTCTTCATTGCCCA \\
\hline MAGEA3 & $\begin{array}{l}\text { F: GGAGCACTGAAGGAGAAGATCTG } \\
\text { R: TGACTCTGGTCAGGGCAACA }\end{array}$ & 142 & TGGGTCTCCATTGCCCAGCTCC \\
\hline NY-ESO-1 & $\begin{array}{l}\text { F: TGCTTGAGTTCTACCTCGCCA } \\
\text { R: TATGTTGCCGGACACAGTGAA }\end{array}$ & 137 & AG GATGCCCCACCGCTTCCC \\
\hline SPANXA1 & $\begin{array}{l}\text { F: GCCACTGACATTGAAGAACCAA } \\
\text { R: GGGTCTCCGGCATCATCTC }\end{array}$ & 115 & ATACAATGGACAAACAATCCAGTGCCGG \\
\hline SPANXC & $\begin{array}{l}\text { F: CCGCCTGCTTCAGTTGC } \\
\text { R: CTGCAGCAGTCAGTCGGATA }\end{array}$ & 167 & TCTGAGTCCTCGACCATACTAGTGGTTCGC \\
\hline SSX1 & $\begin{array}{l}\text { F: GTCACCAACTGCTGCCAAC } \\
\text { R: TTGCTTCTCTTCTCTGATGCTTT }\end{array}$ & 153 & TGCCATGAACGGAGACGACACCTTT \\
\hline PLAC1 & $\begin{array}{l}\text { F: CAAGTTCCTTCTTCCCTTTGGAA } \\
\text { R: CGTCCAGTGAGGATTTCTTCTTC }\end{array}$ & 120 & CAGCTGCCTTCACCAGTGAGCACAA \\
\hline GAPDH & $\begin{array}{l}\text { F: GAAGGTGAAGGTCGGAGTC } \\
\text { R: GAAGATGGTGATGGGATTTC }\end{array}$ & 226 & CAAGCTTCCCGTTCTCAGCC \\
\hline
\end{tabular}

Bp, base pairs; F, forward; FATE1, fetal and adult testis-expressed 1; LAGE1, L antigen family 1; MAGE, melanoma-associated antigen; PLAC1, placenta-specific antigen 1; R, reverse; SPANX, sperm protein associated with the nucleus on the X chromosome; SSX1, synovial sarcoma X breakpoint 1.

along with the 10 healthy donors as control. Cells $\left(\sim 1 \times 10^{7}\right)$ were washed with PBS and incubated with anti-CD45 antibody-coated immunomagnetic microbeads (Miltenyi Biotec $\mathrm{GmbH}$; Bergisch Gladbach, Germany) at $4^{\circ} \mathrm{C}$ for $15 \mathrm{~min}$. The cells were subsequently separated on an autoMACS Column (Miltenyi Biotec $\mathrm{GmbH}$ ) using the sensitive depletion mode for gentle separation to ensure cellular integrity. The CD45-negative fraction, which is effectively enriched in HCC tumor cells due to the depletion of PBMCs, was eluted and cells were collected onto slides using a cytospin device; slides were air dried before being stored at $-20^{\circ} \mathrm{C}$ until required.

Immunocytochemistry of cytokeratin to detect HCC cells in enriched PBMC samples. Slides were fixed in $4 \%$ paraformaldehyde for $20 \mathrm{~min}$ at room temperature and examined for the presence of cytokeratin-positive cells by immunocytochemistry. Following permeabilization with $0.3 \%$ Triton-X-100 for $10 \mathrm{~min}$ at room temperature, and treatment with antigen retrieval solution for $30 \mathrm{~min}$ at $80^{\circ} \mathrm{C}$ (Beyotime Institute of Biotechnology, Haimen, China), the slides were incubated in $3 \% \mathrm{H}_{2} \mathrm{O}_{2}$ for $20 \mathrm{~min}$ and rinsed with distilled water and PBS. Blocking was performed using 5\% normal goat serum (Shanghai Yanjin Biotechnology Co., Ltd., Shanghai, China) at room temperature for $15 \mathrm{~min}$. This was followed by incubation with the mouse anti-cytokeratin, clone CK3-6H5 primary antibody (1:50; cat. no. 130-098-802; Miltenyi Biotec GmbH) overnight at $4^{\circ} \mathrm{C}$. The next day, the slides were rinsed and incubated with the biotinylated horse anti-mouse immunoglobulin G (1:500; cat. no. orb153695; Biorbyt, Ltd., Cambridge, UK) at room temperature for $30 \mathrm{~min}$. The signal was visualized with peroxidase-conjugated streptavidin and 3,3'-diaminobenzidine (DAB). The slides were subsequently examined and photographed using an Olympus BX53 fluorescence microscope (Olympus Corporation, Tokyo, Japan). DAB staining was analyzed by Image-Pro Plus 6.0 software (Media Cybernetics, Inc., Rockville, MD, USA). Unenriched QGY7703 cells were used as the positive control; healthy PBMCs were used as the negative control.

Hematoxylin and eosin (H\&E) staining of PBMC samples enriched in HCC tumor cells. Slides were fixed in $4 \%$ paraformaldehyde and stained with $\mathrm{H} \& \mathrm{E}$ according to routine protocols at room temperature $\left(\sim 25^{\circ} \mathrm{C}\right)$. Briefly, slides were washed with distilled water and stained with hematoxylin solution for 5-10 min, followed by soaking the stained slides in distilled water for $10 \mathrm{~min}$ to remove excess dye. Subsequently, after soaking in $95 \%$ ethanol for $5 \mathrm{sec}$, slides were stained with eosin staining solution for $2 \mathrm{~min}$, which was followed by dehydration with graded alcohol and clearing in xylene. Finally, slides were sealed with neutral gum. The mounted slides were 

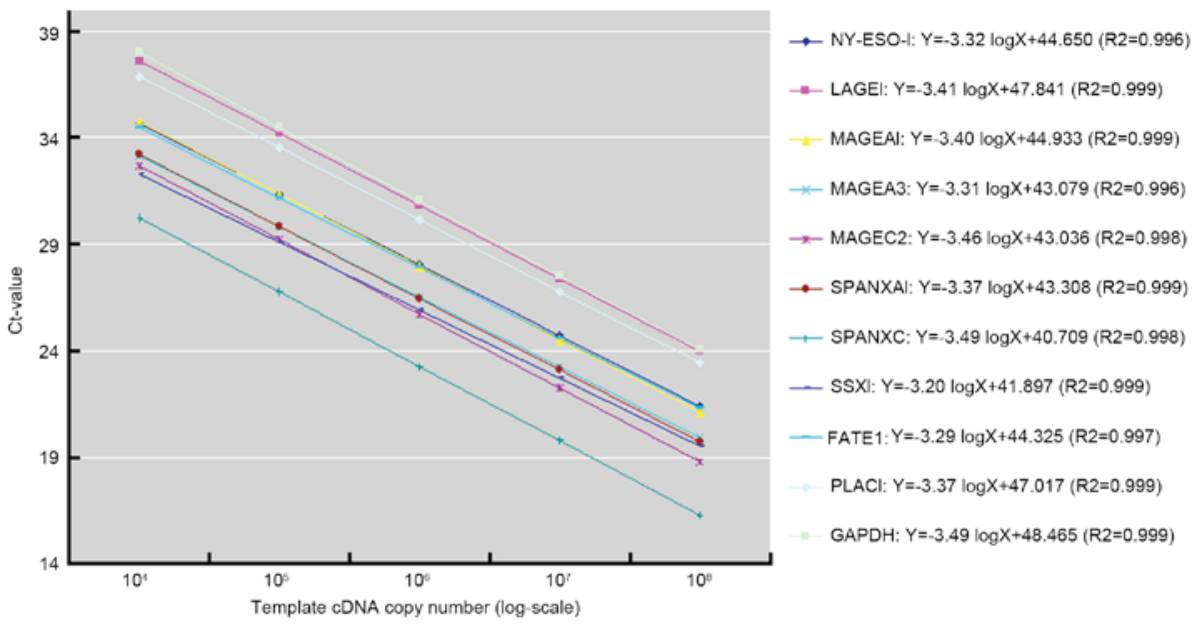

Figure 1. Standard plots (cycle threshold vs. plasmid copy number) for 10 circulating tumor cell markers, including nine cancer-testis antigens and PLAC1 cDNAs. The similar slopes confirm the reproducibility and specificity of reverse transcription-quantitative polymerase chain reaction for detecting circulating tumor cells in the peripheral blood from patients with hepatocellular carcinoma. GAPDH was used as a reference marker. FATE1, fetal and adult testis-expressed 1; LAGE1, L antigen family 1; MAGE, melanoma-associated antigen; PLAC1, placenta-specific antigen 1; SPANX, sperm protein associated with the nucleus on the X chromosome; SSX1, synovial sarcoma X breakpoint 1.
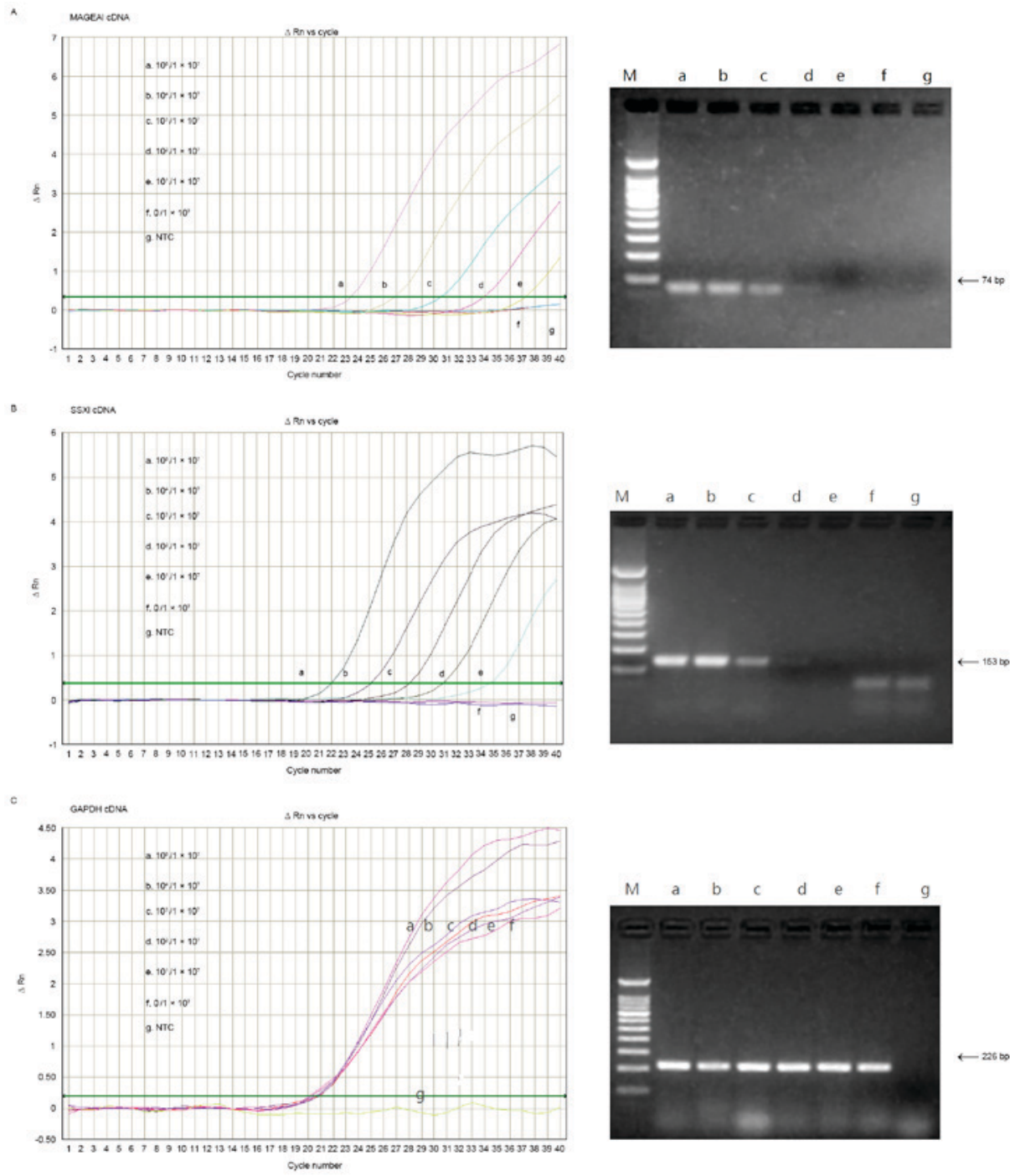

Figure 2. Expression of MAGEA1 and SSX1 mRNAs in different ratios of QGY7703 hepatocellular carcinoma cells and control peripheral blood mononuclear cells were assessed by RT-qPCR (left-hand panels) and standard RT-PCR (right-hand panels) for mRNA expression levels of (A) MAGEA1 and (B) SSX1. (C) GAPDH was used as a reference marker. M, 100 bp marker ladder; MAGEA1, melanoma-associated antigen A1; NTC, negative control for PCR; SSX1, synovial sarcoma X breakpoint 1; RT-qPCR, reverse transcription-quantitative polymerase chain reaction; $\Delta \mathrm{Rn}$, normalized reporter value. 


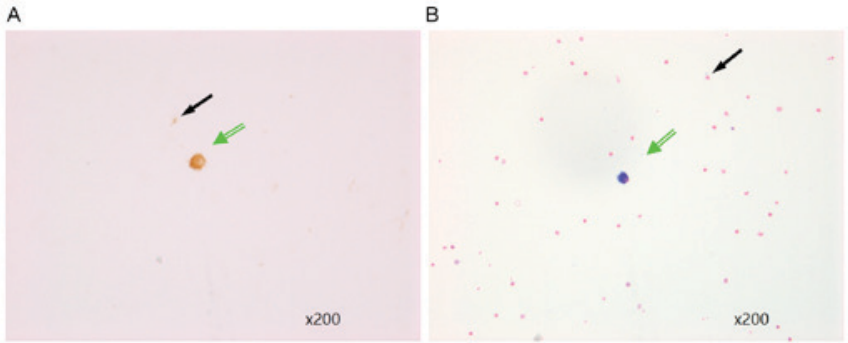

c

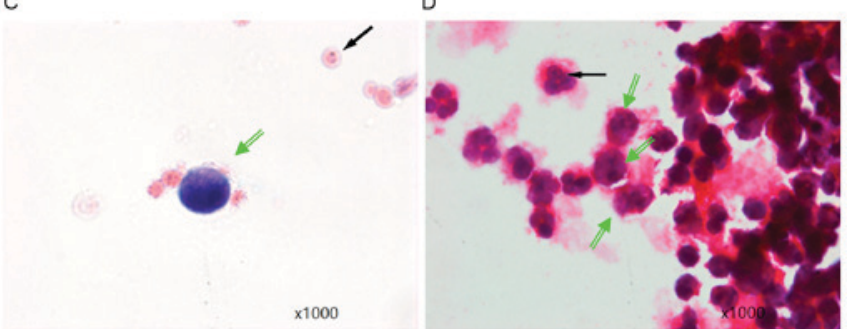

Figure 3. Visualization of HCC cells in peripheral blood samples enriched for HCC cells by magnetic-activated cell sorting. (A) Immunocytochemistry staining of a cytokeratin-positive tumor cell (green arrow) and leukocytes (black arrow) in peripheral blood from a patient with HCC. Magnification, x200. H\&E staining at magnifications of (B) x200 and (C) x1,000 demonstrating tumor cells characterized by large, dark blue and heterogeneously stained nuclei with scant cytoplasm around the nuclei (green arrow). The sizes of the nuclei are approximately five times greater than those of leukocytes (black arrow). (D) H\&E staining demonstrating that HCC tumor cells (green arrows), characterized by large, heterogeneously stained nuclei, are clearly different from other leukocytes (black arrow). Magnification, x1,000. $\mathrm{H} \& \mathrm{E}$, hematoxylin and eosin; HCC, hepatocellular carcinoma.

examined and photographed using an Olympus BX53 fluorescence microscope (Olympus Corporation). Cell morphology was assessed by a chief pathologist who was blinded to the study. Unenriched QGY7703 cells were used as the positive control and healthy PBMCs were used as the negative control. Experiments were repeated three times.

\section{Results}

Standard curves for RT-qPCR. Standard curves for the nine CTAs, PLAC1 and GAPDH cDNAs were constructed using gene-specific recombinant plasmids as copy number standards (Fig. 1). The reducible amplification curves of the 11 standard curves exhibited similar slopes (between -3.20 and -3.49), confirming that the RT-qPCR protocol had acceptable reproducibility and specificity for the detection of the nine CTAs and PLAC1. However, the y-axis intercepts (cycle thresholds) ranged between 40.71 and 48.47 , indicating variations in amplification sensitivities and efficiencies between the different cDNAs.

Sensitivity of the RT-qPCR assay. Using defined ratios of QGY7703 cells and normal PBMCs, the present study was able to detect as few as 10 CTCs among $10^{7}$ PBMCs using RT-qPCR to measure the expression levels of SSX1 and MAGEA1 cDNA in HCC cells (Fig. 2). In contrast, standard RT-PCR was only able to detect as low as $10^{3}$ CTCs in $10^{7}$ PBMCs. This result demonstrated that RT-qPCR was $\sim 100 \mathrm{X}$ more sensitive than RT-PCR for the detection of CTCs through CTA cDNA expression analysis in PBMCs.
Expression rates of CTA and PLAC1 cDNAs in peripheral blood samples from patients with $H C C$. The overall positive detection rate for the 10 tested cDNAs (nine CTAs and PLAC1) in PBMC samples from 51 patients with $\mathrm{HCC}$ was $70.6 \%$ (36/51 patients detected positive for $\geq 1$ target cDNA). However, most of the patients only exhibited between 1 and 3 of these 10 markers. By contrast, none of the tested cDNAs were detected in PBMC samples from the 10 patients with cirrhosis and the 20 healthy patients. The individual positive detection rates were as follows: MAGEA3, 43.1\% (22/51); SSX1, 39.2\% (20/51); MAGEA1, 25.5\% (13/51); NY-ESO-1, 19.6\% (10/51); PLAC1, $19.6 \%$ (10/51); LAGE1, 17.6\% (9/51); MAGEC2, 7.8\% (4/51); SPANXC, 7.8\% (4/51); SPANXA1, $2.0 \%$ (1/51); and FATE1, $0 \%(0 / 51)$. Six of the genes (MAGEA3, SSX1, MAGEA1, NY-ESO-1, PLAC1 and LAGE1) demonstrated high positive rates in the PBMCs of patients with HCC and were selected for further analysis. The expression levels of these six cDNAs, relative to GAPDH, were as follows: MAGEA3, $1 / 10^{3}-1 / 10^{7}$; SSX1, 1/10 $3.5-1 / 10^{6.5}$; MAGEA1, 1/10 $2.5-1 / 10^{6.5}$; NY-ESO-1, $1 / 10^{3.5}-1 / 10^{6}$; PLAC1, $1 / 10^{4}-1 / 10^{5.5}$; and LAGE1, $1 / 10^{2}-1 / 10^{5.5}$.

HCC cell enrichment in PBMC and detection by immunocytochemistry and $H \& E$ staining. A total of 19 PBMC samples that tested positive for CTA and PLAC1 cDNAs by RT-qPCR were randomly selected for tumor cell enrichment through the depletion of leukocytes by MACS. H\&E staining and immunocytochemistry with an anti-cytokeratin antibody were used to detect HCC cells. A total of 12/19 (63.2\%) samples exhibited intact tumor cells (Fig. 3), of which HCC cells were identified using both techniques (10/12 samples) or were only detected using one of the two techniques (2/12 samples). By contrast, HCC cells were not observed by either technique in PBMC samples from patients with cirrhosis or normal individuals.

\section{Discussion}

CTCs contribute to recurrence and metastasis and are often an indication of advanced stage cancer and poor prognosis (15). Recent reports $(10,12)$ have demonstrated that by examining CTA expression levels, CTCs may be detected in the blood with greater specificity compared with traditional techniques, such as AFP evaluation, RT-PCR or nested-primer RT-PCR. To determine whether RT-qPCR was able to detect CTCs in patients with $\mathrm{HCC}$ with greater sensitivity and specificity than standard RT-PCR, the present study evaluated the expression levels of 10 CTC marker cDNAs (nine CTAs and PLAC1) by RT-qPCR in blood samples collected from 51 preoperative patients with HCC. In addition, anti-CD45 antibody coated immunomagnetic beads were used to enrich PBMC samples for tumor cells to establish the potential value of this technique for prognostic evaluation of patients with HCC.

Results from the present study revealed that RT-qPCR of CTA cDNAs was able to detect 10 tumor cells per $10^{7}$ PBMCs, demonstrating that this technique was $\sim 100 \mathrm{X}$ more sensitive than standard RT-PCR for the detection of CTCs. Of the 10 tested tumor markers, MAGEA3, SSX1, MAGEA1, NY-ESO-1, PLAC1 and LAGE1 cDNAs had positive detection rates $>10 \%$; the overall positive detection rate was $70.6 \%$. However, the expression levels of these six cDNAs varied among the 51 patients with HCC that were examined; most patients 
tested positive for only 1-3 of the 10 CTC marker cDNAs. In contrast, all patients with cirrhosis and healthy individuals that were examined tested negative for the $10 \mathrm{cDNAs}$.

Enrichment of tumor cells by MACS, combined with H\&E staining and morphological assessment, additionally emerged as a potential method for detecting CTCs in patients with HCC; positive identification of intact tumor cells was achieved in $63.2 \%(12 / 19)$ of patients that had been identified as positive by RT-qPCR. This result suggested that the two techniques may be combined as a valuable prognostic tool; however, comparable studies on alternative methods for identifying intact tumor cells in HCC are limited.

In conclusion, the present study demonstrated that the use of RT-qPCR to evaluate the expression rates of CTAs and PLAC1 in PBMC samples may offer a highly sensitive and specific technique to detect CTCs in the peripheral blood of patients with HCC. Furthermore, the accuracy of this method may be enhanced by MACS tumor cell enrichment.

\section{Acknowledgements}

The present study was supported by the Navigating Plan of Research Fund of Beijing Ditan Hospital, Capital Medical University (grant no. DTQH201405) and the Beijing Municipal Administration of Hospitals Incubating Program (grant no. PX2016043).

\section{References}

1. Parkin DM, Bray F, Ferlay J and Pisani P: Global cancer statistics, 2002. CA Cancer J Clin 55: 74-108, 2005.

2. Parkin DM: Global cancer statistics in the year 2000. Lancet Oncol 2: 533-543, 2001.

3. Elshimali YI and Grody WW: The clinical significance of circulating tumor cells in the peripheral blood. Diagn Mol Pathol 15: 187-194, 2006
4. Hosokawa M, Hayata T, Fukuda Y, Arakaki A, Yoshino T, Tanaka $\mathrm{T}$ and Matsunaga T: Size-selective microcavity array for rapid and efficient detection of circulating tumor cells. Anal Chem 82: 6629-6635, 2010

5. Samija I, Lukac J, Kusić Z, Situm M and Samija M: Detection of disseminated melanoma cells by reverse-transcriptionpolymerase chain reaction. Coll Antropol 31: 1187-1194, 2007.

6. Schefe JH, Lehmann KE, Buschmann IR, Unger T and Funke-Kaiser H: Quantitative real-time RT-PCR data analysis: Current concepts and the novel 'gene expression's CT difference' formula. J Mol Med (Berl) 84: 901-910, 2006.

7. Morimoto O, Nagano H, Miyamoto A, Fujiwara Y, Kondo M, Yamamoto T, Ota H, Nakamura M, Wada H, Damdinsuren B, et al: Association between recurrence of hepatocellular carcinoma and alpha-fetoprotein messenger RNA levels in peripheral blood. Surg Today 35: 1033-1041, 2005.

8. Yao F, Guo JM, Xu CF, Lou YL, Xiao BX, Zhou WH, Chen J, Hu YR, Liu Z and Hong GF: Detecting AFP mRNA in peripheral blood of the patients with hepatocellular carcinoma, liver cirrhosis and hepatitis. Clin Chim Acta 361: 119-127, 2005.

9. Lu Y, Wu LQ, LüZH, Wang XJ and Yang JY: Expression of SSX-1 and NY-ESO-1 mRNA in tumor tissues and its corresponding peripheral blood expression in patients with hepatocellular carcinoma. Chin Med J (Engl) 120: 1042-1046, 2007.

10. Mou DC, Cai SL, Peng JR, Wang Y, Chen HS, Pang XW, Leng XS and Chen WF: Evaluation of MAGE-1 and MAGE-3 as tumour-specific markers to detect blood dissemination of hepatocellular carcinoma cells. Br J Cancer 86: 110-116, 2002.

11. Zendman AJ, Ruiter DJ and Van Muijen GN: Cancer/testisassociated genes: Identification, expression profile, and putative function. J Cell Physiol 194: 272-288, 2003.

12. Peng JR, Chen HS, Mou DC, Cao J, Cong X, Qin LL, Wei L, Leng XS, Wang Y and Chen WF: Expression of cancer/testis (CT) antigens in Chinese hepatocellular carcinoma and its correlation with clinical parameters. Cancer Lett 219: 223-232, 2005.

13. Koslowski M, Sahin U, Mitnacht-Kraus R, Seitz G, Huber C and Türeci O: A placenta-specific gene ectopically activated in many human cancers is essentially involved in malignant cell processes. Cancer Res 67: 9528-9534, 2007.

14. Bustin SA: Absolute quantification of mRNA using real-time reverse transcription polymerase chain reaction assays. J Mol Endocrinol 25: 169-193, 2000.

15. Walzer N and Kulik LM: Hepatocellular carcinoma: Latest developments. Curr Opin Gastroenterol 24: 312-319, 2008. 